\title{
“CÓMO VA LA COSA?" \\ O RESTRICCIONES DE USO EN LOS SALUDOS DE FINGIMIENTO FÓRICO
}

\author{
Mario Hernández Delgado*
}

\begin{abstract}
RESUMEN
En primer lugar, y con base en los datos obtenidos en un cuestionario, se verifica que en el medio costarricense existe una clase especial de saludos que recurren a la inclusión de elementos que, a pesar de tener normalmente una interpretación fórica, en estos casos carecen del anclaje textual o referencial necesario, razón por la cual en este trabajo denomino saludos de fingimiento fórico, o seudofóricos, a los que presentan esta peculiaridad. Entre ellos se cuentan: “Cómo va la cosa?”, “Cómo va eso?”, “Así es...!”, “¡Así es la cosa!”, “¿Eso!” y otros que comparten la misma característica. Por otro lado, y también según los resultados del cuestionario, se muestra que estos saludos presentan restricciones de uso, y que estas tienen que ver centralmente con la distancia social. Finalmente, se argumenta que las restricciones de uso de este tipo de enunciados están directamente relacionadas con el hecho de que en ellos se recurra a ficciones fóricas. Palabras clave: Cortesía, saludos, deixis, enunciados fóricos, artículo (definido), distancia social.
\end{abstract}

\begin{abstract}
Firstly and based on the data obtained from a questionnaire it has been proven that in Costa cases they lack the necessary anchorage, either textual or referential. This is the reason why in this paper I call the ones with this characteristic phoric pretence greetings or pseudophoric greetings. Among them we have: “¿Cómo va la cosa?”, “Cómo va eso?”, “¡Así es...!”, “¡Así es la cosa!”, “¿Eso!” and other ones that share the same feature. On the other hand and also according to the results of the questionnaire it is proven that these greetings have usage restrictions and that these restrictions have to do with social distance. Finally, it is argued that the usage restrictions of these kind of utterances are directly related with the fact that they use phoric fictions.
\end{abstract}

Key Words: Politeness, greetings, deixis, phoric utterances, (definite) article, social distance. 


\section{Introducción}

Lo que se presenta a continuación corresponde a la comunicación expuesta en el marco de las II Jornadas de Investigación Lexicográfica, organizadas por el Programa de Lexicografía (ELEXHICÓS) del Instituto de Investigaciones Lingüísticas de la Universidad de Costa Rica. Como en esa ocasión, debo aclarar en esta memoria que el texto que sigue consiste en un avance de una investigación en proceso, y que, una vez que se haya completado el estudio, se dará a conocer una versión más acabada que contendrá más elementos, referencias y argumentos que los aquí incluidos.

La comunicación que se presentó en la mencionada actividad académica llevó por nombre sencillamente “Cómo va la cosa?", pero, para su publicación en este medio, decidí ampliar el título para efectos de que sea más descriptivo. De este modo, desde un inicio quedará claro para cualquier lector con qué valor serán tratadas aquí esa y las otras formulaciones que luego se indicarán. En síntesis, serán estudiadas con su valor de saludos (en el medio costarricense).

Respecto al carácter y función de los saludos en general, en primer lugar hay que considerar que esencialmente constituyen un tipo de actos mediante los cuales se concreta el reconocimiento interaccional del otro. En otras palabras, sirven primariamente para dar a entender al otro que se reconoce su presencia (es decir, su "estar ahí") como un potencial interlocutor.

Ahora bien, dado que con el empleo de determinados gestos es perfectamente posible dar a entender al otro que se reconoce su presencia como posible interlocutor, vamos a señalar brevemente cuáles son las principales características específicas del saludo verbal, para lo cual voy a seguir a Haverkate (1994):

a. En primer lugar, y en la medida en que tiene efectos sobre la imagen social del otro, se debe considerar como un acto de cortesía verbal y, por lo tanto, adquiere sentido en un marco más amplio de producción e interpretación de la conducta social.

b. Su realización formal está basada en fórmulas rutinarias, generalmente fosilizadas y con una estructura simple, como hola, ¿qué tal?, ¿cómo te va? (cf. Haverkate, op. cit.: 84).

c. Las condiciones de uso e interpretación de los enunciados utilizados para saludar son estrictamente convencionales. Por ejemplo, cuando estos tienen forma de pregunta: “(...) estas preguntas se interpretan ante todo en un sentido simbólico; no se suele esperar una respuesta concreta a las mismas (...)" (Haverkate, op. cit.: 85) 1 .

d. El saludo está desprovisto de valor conceptual o descriptivo. En otras palabras: “(...) el acto de saludar no sirve para transmitir información proposicional" (Haverkate, op. cit.: 84). La causa de esto es que los saludos “(...) no se intercambian para expresar una descripción del mundo extralingüístico" (ibid.).

e. Los saludos “(...) constituyen una clase típicamente cerrada" (Haverkate, op. cit.: 85). Es decir, la aceptación de nuevas fórmulas de saludo ocurre de forma lenta y poco frecuente, por lo que el paradigma de los saludos en general tiene una cantidad estable de miembros.

Adicionalmente, Haverkate (op. cit.: 85) señala las que siguen como las principales funciones interactivas del saludo:

a. “(...) sirve para abrir el canal comunicativo; es decir, funciona como señal para llamar la atención del interlocutor, incitándole a participar en un intercambio verbal".

b. “(...) puede contribuir a evitar que se produzca una tensión social, provocada cuando dos personas se encuentran en una situación comunicativa potencial sin cruzar palabra (...)".

c. “(...) de acuerdo con la fórmula seleccionada, el saludo sirve para 
establecer o confirmar una determinada relación interaccional, tal como se define por factores como posición social, grado de intimidad y afecto".

\section{El objeto de estudio}

Volviendo al conjunto de expresiones que mencioné inicialmente como objeto de este estudio, y del cual la que aparece en el título de este escrito es representante, son las que aparecen en el siguiente cuadro:

\section{CUADRO 1}

Muestra de la clase de expresiones que serán objeto de estudio

\begin{tabular}{l}
\hline 1 - ¿Cómo va la cosa? \\
\hline 2 - ¿Cómo va la vara? \\
\hline 3 - ¡Cómo va eso? \\
\hline 4 - ¡Ahí está el hombre (el amigo / el compañero)...! \\
\hline 5 - ¡Ahí está la mujer (la amiga / la compañera)...! \\
\hline 6 - ¡Así es! \\
\hline 7 - ¡Así es la cosa! \\
\hline 8 - ¡Así es la vara! \\
\hline 9 - ¡Eso es! \\
\hline 10 - ¡Eso! \\
\hline 11 - ¡Eso eso! \\
\hline
\end{tabular}

\section{Objetivos}

Los objetivos de este estudio son:

i. Mostrar que las expresiones del cuadro 1 existen como saludos en el medio costarricense, o, en otras palabras, que son utilizadas al menos por algunos costarricenses con ese valor.

ii. Mostrar que todos los saludos del cuadro 1 tienen al menos una característica formal en común, con base en la cual se pueden establecer como una clase, es decir, como un conjunto para el cual se puede operacionalizar un criterio de membresía.

iii. Mostrar que los saludos del cuadro 1 presentan restricciones de uso, y que estas están directamente vinculadas con la interpretación ficcional de sus componentes fóricos.

En lo que sigue, voy a hacer el desarrollo de la temática de la exposición. La forma en que está organizada consiste en ir declarando nuevamente los objetivos, uno a uno, e ir dando los argumentos tendientes a dar cada uno por satisfecho antes de pasar al siguiente.

\section{Primer objetivo}

El primer objetivo es, según se indicó, mostrar que las expresiones del cuadro 1 "existen" como saludos en el medio costarricense; es decir, que son utilizadas al menos por algunos costarricenses con ese valor.

Para validar la suposición de que todas esas expresiones realmente se utilizan como saludos, opté por la confección de un cuestionario $^{2}$, y lo aquí tratado tendrá, pues, cualquier limitación que se considere intrínseca a los datos basados en opinión. También debo aclarar, respecto de los datos obtenidos, que su tratamiento será fundamentalmente cualitativo e instrumental, y que en ningún momento se tendrá la pretensión de que los resultados numéricos sean generalizables ni, mucho menos, estadísticamente proyectables. Sin embargo, como se verá en su momento, para los objetivos de este trabajo la información así obtenida será suficientemente útil.

En cuanto a quienes accedieron a llenar el cuestionario, fueron 20 personas (10 hombres y 10 mujeres) que actualmente tienen, o en el pasado han tenido, algún vínculo con la Universidad de Costa Rica, ya sea como funcionarios, docentes o estudiantes. En el cuadro 2 se pueden observar los datos relativos al conocimiento y uso de las formulaciones en estudio por parte de los informantes: 
CUADRO 2

Reconocimiento y uso como saludos de las expresiones bajo estudio

\begin{tabular}{|c|c|c|c|c|c|c|}
\hline \multirow[t]{2}{*}{ Expresión } & \multicolumn{3}{|c|}{ La conocen como saludo } & \multicolumn{3}{|c|}{ La usan como saludo } \\
\hline & Total & Mujeres & Hombres & Total & Mujeres & Hombres \\
\hline $\begin{array}{l}1-\text { ¿Cómo va la } \\
\text { cosa? }\end{array}$ & 19 & 10 & 9 & 11 & 5 & 6 \\
\hline $\begin{array}{l}2-\text { ¿Cómo va la } \\
\text { vara? }\end{array}$ & 18 & 10 & 8 & 6 & 4 & 2 \\
\hline 3 - ¿Cómo va eso? & 19 & 9 & 10 & 7 & 2 & 5 \\
\hline $\begin{array}{l}4 \text { - ¡Ahí está el } \\
\text { hombre (el amigo / } \\
\text { el compañero)...! }\end{array}$ & 18 & 9 & 9 & 5 & 0 & 5 \\
\hline $\begin{array}{l}5 \text { - ¡Ahí está la } \\
\text { mujer (la amiga / la } \\
\text { compañera)...! }\end{array}$ & 7 & 5 & 2 & 0 & 0 & 0 \\
\hline 6 - ¡Así es! & 14 & 6 & 8 & 6 & 1 & 5 \\
\hline 7 - ¡Así es la cosa! & 14 & 7 & 7 & 7 & 1 & 6 \\
\hline 8 - ¡Así es la vara! & 13 & 6 & 7 & 5 & 1 & 4 \\
\hline $9-$ ¡Eso es! & 15 & 8 & 7 & 7 & 2 & 5 \\
\hline $10-¡ ¡ E s o !$ & 19 & 9 & 10 & 13 & 6 & 7 \\
\hline $11-$ ¡Eso eso! & 19 & 9 & 10 & 8 & 3 & 5 \\
\hline
\end{tabular}

Como se puede observar, todas las expresiones fueron reconocidas por al menos algunas de las personas; en otras palabras, todas han sido, como mínimo, escuchadas a otras personas en función de saludos. Además, las cantidades de mujeres y de hombres que indicaron familiaridad con las diversas fórmulas en general no muestran diferencias amplias. Aun cuando al pasar a verificar el uso efectivo observamos que se presenta un decaimiento notable en las cifras, es posible afirmar sin problemas que, con excepción de la formulación 5 , todas las demás sirven o en algún momento han servido a algunos de los integrantes de la muestra para saludar.

Otra información complementaria que se logró obtener se relaciona con la frecuencia de uso de esos saludos por parte de los informantes. En el cuadro 3 se presentan las cifras:

Como se puede observar, la frecuencia de uso, según el criterio de opinión de quienes manifestaron utilizar esos saludos, en términos
CUADRO 3

Frecuencia de uso de los saludos bajo estudio

\begin{tabular}{|c|c|c|c|}
\hline \multirow[t]{2}{*}{ Expresión } & \multicolumn{3}{|c|}{ Frecuencia de uso } \\
\hline & $\begin{array}{l}\text { Casi } \\
\text { nunca }\end{array}$ & $\begin{array}{l}\text { Algunas } \\
\text { veces }\end{array}$ & Muchas veces \\
\hline 1 - ¿Cómo va la cosa? & 6 & 5 & 0 \\
\hline 2 - ¿Cómo va la vara? & 4 & 2 & 0 \\
\hline 3 - ¿Cómo va eso? & 3 & 4 & 0 \\
\hline $\begin{array}{l}4 \text { - ¡Ahí está el } \\
\text { hombre (el amigo / el } \\
\text { compañero)...! }\end{array}$ & 2 & 3 & 0 \\
\hline $\begin{array}{l}5 \text { - ¡Ahí está la mujer } \\
\text { (la amiga / la compa- } \\
\text { ñera)...! }\end{array}$ & NA & NA & NA \\
\hline 6 - ¡Así es! & 2 & 4 & 0 \\
\hline 7 - ¡Así es la cosa! & 2 & 4 & 1 \\
\hline 8 - ¡Así es la vara! & 1 & 3 & 1 \\
\hline 9 - ¡Eso es! & 1 & 5 & 1 \\
\hline 10 - ¡Eso! & 7 & 4 & 2 \\
\hline 11 - ¡Eso eso! & 5 & 2 & 1 \\
\hline
\end{tabular}


generales se mueve entre "casi nunca" (es decir, muy pocas veces) y "algunas veces". De este dato, la conclusión que interesa es que, aunque tienen usuarios, estos saludos no tienden a ser muy utilizados, de modo que ya la frecuencia de uso presenta un carácter restrictivo.

\section{Segundo objetivo}

Pasando ahora al segundo objetivo de este trabajo, consiste en lo siguiente: mostrar que todos los saludos del cuadro 1 tienen al menos una característica formal en común, con base en la cual se pueden establecer como una clase, es decir, como un conjunto para el cual se puede operacionalizar un criterio de membresía. Para visualizar mejor esa característica o criterio de membresía, consideremos (cuadro 4) una ligera reordenación del cuadro 1 en la cual, además, están resaltados (en negrita a la vez que en cursiva) los elementos que interesan:

CUADRO 4

Elementos comunes de los saludos bajo estudio para su consideración como clase

\begin{tabular}{|c|}
\hline 1 - ¿Cómo va la cosa? \\
\hline 2 - ¿Cómo va $\boldsymbol{l a}$ vara? \\
\hline $\begin{array}{l}3 \text { - ¡Ahí está } \boldsymbol{e l} \text { hombre (el amigo / el compañe- } \\
\text { ro)...! }\end{array}$ \\
\hline 4 - ¡Ahí está $\boldsymbol{l a}$ mujer ( $\boldsymbol{l a}$ amiga / la compañera)...! \\
\hline $5-$ ¡Así es la cosa! \\
\hline 6 - ¡Así es la vara! \\
\hline 7 - ¡Así es! \\
\hline 8 - ¿Cómo va eso? \\
\hline 9 - ¡Eso es! \\
\hline $10-¡$ ¡Eso! \\
\hline $11-¡$ ¡Eso eso! \\
\hline
\end{tabular}

Como se puede notar por los elementos resaltados en el cuadro 4, todas estas formulaciones incluyen componentes "mostrativos" u "ostensivos" (como son el pronombre demostrativo eso, el adverbio de modo así y el de lugar $a h i)^{3}$, frases nominales introducidas por artículos definidos $(e l, l a)$ o ambos.

El punto que interesa aquí es que todos esos elementos lingüísticos -artículos definidos y (de)mostrativos- se pueden conjuntar bajo la categoría de elementos fóricos o elementos de referencia fórica.

Para ver esto, comencemos por definir la referencia fórica como el fenómeno lingüístico en el que un elemento de un enunciado remite a información externa a ese enunciado, ya sea que esa información se encuentre en el contexto lingüístico - esto es, el texto o discurso que precede o sigue a tal enunciado, concepto que algunos llaman cotexto- o ya sea que se encuentre en la situación de habla, para la adecuada identificación del referente al que apunta ese elemento o al que apunta otro elemento al que este acompaña y determina.

Con base en esa caracterización, el pronombre eso, que aparece en nuestro corpus (y, en general, los pronombres/adjetivos demostrativos), el adverbio ahi (y muchos otros adverbios de lugar, además de muchos de los de tiempo) y el adverbio así (y otros adverbios de modo), se deben considerar elementos fóricos, ya que, para que adquieran sentido pleno, se requiere información externa al enunciado, sea que esta provenga de la situación extralingüística en la que se realiza el acto de enunciación (fenómeno llamado de referencia exofórica) o que provenga del entorno discursivo (fenómeno conocido como de referencia endofórica). Por otro lado, la referencia endofórica puede ser de dos tipos: anafórica, si el elemento fórico remite a una información que se dio en el discurso anterior, o catafórica, si esa información se encuentra en el discurso que vendrá.

Como en este punto ya se habrá notado, este fenómeno se tiende a asociar típicamente con el que se conoce bajo el nombre de deixis, respecto del cual Levinson (1983/1989: 54) indica lo siguiente:

Esencialmente, la deixis tiene que ver con los recursos que utilizan las lenguas para codificar o 
gramaticalizar rasgos del contexto de enunciación o del evento de habla, de modo que también tiene que ver con las formas en que la interpretación de los enunciados depende del análisis de ese contexto de enunciación. [Las negritas aparecen en el original].

Aunque las categorías que más tradicionalmente se contaban como deícticas eran la de lugar (p. e., aquí, eso, aquella), la de tiempo (como mañana, dentro de una hora, después, el año entrante) y la de persona (que típicamente incluye los pronombres personales y las flexiones gramaticales también de persona), Levinson (1983/1989: 62 y 85-94) siguiendo a Lyons y a Fillmore, agrega la de deixis social (como las formas de tratamiento y las alternancias de cercanía/distancia social en los pronombres personales) y la de deixis discursiva o textual.

Esta última, la deixis discursiva, consistiría fundamentalmente en el empleo de deícticos espaciales, y en algunos casos de deícticos temporales, para remitir a información presente en el entorno discursivo, por lo que su rasgo más notable es su carácter eminentemente endofórico.

Ahora bien, en el caso del artículo definido, aunque la inclusión de esta categoría como un recurso de señalización deíctica no siempre se acepta sin reservas, en realidad muchas de sus funciones efectivamente parecen coincidir con las de los elementos típicamente deícticos. En cuando a esto, Rodríguez-Izquierdo y Gavala (1976: 123-124), señala que:

La anáfora que ejerce el artículo ha de entenderse que es una deixis hacia un contexto (...). La señalización que supone esta deixis puede hacer remitir la referencia de un sustantivo bien a un contexto idiomático, que coincide con la lengua supuestamente conocida por el oyente, bien a un contexto situacional inmediato. En todo caso, se pone en relación el sustantivo con un contexto de conocimientos que el hablante supone debe ser el del oyente.

Esta propiedad del artículo definido es precisamente la que lo constituye en un actualizador de determinado contenido conceptual que se presupone de dominio del oyente. Por otro lado, es también por esa característica relacional que en las gramáticas del texto se considera a tal elemento como un recurso de coherencia textual, en contraposición con las gramáticas tradicionales, en las que interesa fundamentalmente por sus propiedades intraoracionales. Por otro lado, la afinidad del artículo respecto de los elementos prototípicamente deícticos va más allá de la sola deixis endofórica, pues, de hecho, no es difícil imaginar una cantidad virtualmente ilimitada de situaciones en las que, en ausencia de información discursiva que permita identificar un referente que corresponda al nombre al que el artículo acompaña, el oyente buscará ese referente en el entorno situacional inmediato. Tal como se puede constatar en el ejemplo iii (abajo), esto no es más que otro caso de referencia exofórica. Debido a todo lo anterior, consideramos justificado que aquí tratemos al artículo (definido), sin mayor problema, como un señalador de referencia fórica.

A continuación, unos pocos ejemplos de referencia fórica (en cursiva los elementos que interesan):

\section{a. Referencia exofórica:}

a.1. [Un alumno, balanceando con la mano un celular y dirigiéndose a los compañeros que ya están saliendo del aula]:

\section{- ¿Quién dejó esto olvidado?}

a.2. [Un profesor de matemáticas, dirigiéndose a sus alumnos mientras desarrolla en la pizarra la solución de un ejercicio que acababa de escribir en ella]:

- Este problema se resuelve así...

a.3. [Un electricista, dirigiéndose a su ayudante]

- Páseme el alicate.

b. Referencia endofórica (solo se ejemplifica la anafórica):

b.1. A: - Los técnicos encontraron un grave problema en la instalación de la red.

B: - Bueno, en realidad eso no es nuevo, ya había sido reportado. 
b.2. [Un amigo a otro que acaba de darle sus argumentos en favor del Proyecto de Reforma Fiscal]

A:- Todos deberíamos apoyar el Proyecto, porque... [argumentos].

B: - Bueno, perdón, pero ahí hay un grave error de razonamiento...

\section{b.3. [Noticia]}

"Un hombre fue atropellado por $\underline{\text { un }}$ automóvil en San Pedro. El vehículo se dio a la fuga."

Regresando ahora a nuestro pequeño corpus de saludos, y habiendo visto que tienen como característica común la presencia de componentes de referencia fórica, creo que no hay problema en que, por lo menos provisionalmente, los llamemos saludos fóricos para identificarlos como clase.

Ahora bien, a pesar de ese rasgo formal que tienen en común, podemos aún preguntarnos si eso es suficiente para considerarlos expresiones propiamente fóricas. En cuanto a esto, mi posición es que la presencia de esas características formales no es suficiente para darles esa calificación. En otras palabras, que, a pesar de tener la apariencia de expresiones fóricas, no existen las condiciones para una interpretación fórica o deíctica de esas formulaciones utilizadas como saludos. Para fundamentar esta opinión, me remito a los dos indicadores siguientes:

i) En primer lugar, hay que notar que no existen las condiciones objetivas para una interpretación fórica, ya seaintradiscursiva o situacional, ya que, si tomamos en cuenta que, por su carácter fático, el saludo sirve precisamente para introducir o preparar una situación potencialmente conversacional, normalmente no supone una interacción inmediatamente anterior entre los interlocutores de la cual pudiera tomar -o, al menos, la cual pudiera sugerir- el anclaje textual o referencial necesario para que, en nuestro caso, los elementos fóricos adquieran un sentido suficientemente definido. Como apoyo de esto, me remito a Haverkate (1994: 71), quien afirma que: "Como el saludo es un acto cortés desprovisto de valor conceptual, se explica que ocupe la posición más periférica posible, o sea, la posición inicial absoluta del macroacto [de habla, es decir, del acto de habla global que se desarrolla como discurso]".

ii) Un segundo indicador tiene que ver con la incompatibilidad de los saludos que hemos llamado fóricos con respecto a la señalización gestual. Según afirma Cairns (1991: 21): “Todas las expresiones de deixis espacial tienen en común el hecho de que pueden ser acompañadas simultáneamente, aun en el habla adulta, por gestos no verbales de señalamiento". Si a esto unimos la hipótesis compartida por todos los teóricos que Yang (2011) llama localistas (y, a la hipótesis como tal, localismo), de que "las expresiones espaciales son más básicas, gramatical y semánticamente, que varios tipos de expresiones no espaciales ${ }^{4}$ ", no tiene por qué resultar extraño que expresiones deícticas o fóricas no espaciales "hereden" la posibilidad de concurrir, de forma natural, con gestos mostrativos.

Por ejemplo, tomemos en cuenta que, cuando emitimos términos como aquí, ahi, allá, él, aquellos, eso, etc., para referirnos a posiciones o entidades en el espacio, perfectamente podemos hacer la señalización correspondiente. Incluso en el caso de la deixis discursiva (como cuando decimos "ahí está el fallo en el argumento" o "eso es lo mismo que yo pienso") podemos acompañar tales aserciones con una señalización gestual simbólica, como si estuviéramos señalando algo que existe o forma parte del espacio tridimensional. También podemos ver que este acompañamiento gestual es posible incluso en el caso de muchas 
expresiones de carácter temporal, como cuando decimos ya y señalamos como lo haríamos hacia un punto en el espacio cercano (con un gesto idéntico al que podríamos utilizar para acompañar la enunciación de un aquí, por ejemplo), o como cuando decimos "pasaron la reunión para mañana" y hacemos un gesto que imita el movimiento de algo hacia adelante.

Pues bien, en el caso de los saludos fóricos, acompañarlos con un señalamiento gestual sólo produciría confusión en el receptor o, en otro caso, induciría a que este no tome el enunciado del emisor como un saludo, sino como una pregunta "genuina" (es decir, como una petición de información) en caso de que tenga forma interrogativa, o como una aserción referida a alguna entidad que se dé por consabida o que esté presente en el entorno físico, en el caso de que tenga forma declarativa.

La pregunta obligada en este punto es: Pero, entonces, ¿se pueden considerar esos saludos, en última instancia, como enunciados fóricos, o no? Mi posición es que la respuesta no puede ser categóricamente un sí o un no, y, en lo que falta, voy a argumentar por qué creo que una mejor denominación para ellos es la de saludos seudofóricos o saludos de fingimiento fórico. $\mathrm{Y}$, con esto, paso al tercer objetivo:

\section{Tercer objetivo}

El tercer objetivo es, según se estableció antes, mostrar que los saludos del cuadro 1 presentan restricciones de uso, y que estas están directamente relacionadas con la interpretación ficcional de sus componentes fóricos, es decir, con el hecho de que sean saludos seudofóricos o de fingimiento fórico. Ahora bien, como indiqué al inicio, lo aquí informado es esencialmente un avance de una investigación en proceso, debido a lo cual, lo que voy a hacer a continuación es solamente adelantar algunos elementos para que se tenga idea de la línea de razonamiento.

Para esto, en primer lugar voy a hacer referencia a otros de los datos obtenidos en el cuestionario antes mencionado, los cuales se encuentran en el cuadro 5:

CUADRO 5

Grado de familiaridad o confianza que se debe tener con una persona para dirigirle estos saludos

\begin{tabular}{ll}
\hline 1 - ¡Cómo va la cosa? & vecinos, compañeros, amigos, familiares \\
\hline 2 - ¿Cómo va la vara? & compañeros, amigos, amigos íntimos, familiares \\
\hline 3 - ¿Cómo va eso? & conocidos, compañeros, amigos, familiares \\
\hline 4 - ¡Ahí está el hombre (el amigo / el compañero)...! & conocidos, compañeros, amigos, familiares \\
\hline 5 - ¡Ahí está la mujer (la amiga/ la compañera)...! & conocidas, compañeras, amigas \\
\hline 6 - ¡Así es! & $\begin{array}{l}\text { conocidos, compañeros, amigos, amigos cercanos, } \\
\text { familiares }\end{array}$ \\
\hline 7 - ¡Así es la cosa! & $\begin{array}{l}\text { conocidos, compañeros, amigos, amigos cercanos, } \\
\text { familiares }\end{array}$ \\
\hline 8 - ¡Así es la vara! & $\begin{array}{l}\text { conocidos, compañeros, amigos, amigos cercanos o } \\
\text { intimos, familiares }\end{array}$ \\
\hline 9 - ¡Eso es! & $\begin{array}{l}\text { conocidos, compañeros, amistades, amigos, amigos } \\
\text { cercanos, familiares }\end{array}$ \\
\hline 10 - ¡Eso! & $\begin{array}{c}\text { conocidos, compañeros, amigos, amigos } \\
\text { conocidos, compañeros, amigos, amigos cercanos, } \\
\text { familiares }\end{array}$ \\
\hline
\end{tabular}


Como podemos observar en el cuadro 5, al pedir a los informantes que indicaran los tipos de personas a las cuales consideraban apropiado o aceptable dirigir esos saludos, la tendencia es claramente a incluir entre ellos únicamente a personas con las cuales se tiene cierto grado de cercanía o familiaridad (en el sentido de distancia social). De hecho, una informante explícitamente indicó: "Jamás saludaría así a mi jefa". En otro punto del cuestionario, 8 de los informantes valoraron esos saludos como informales (característica diafásica), 3 como propios del uso popular (característica diafásica o tal vez diastrática), una persona como propios de la clase media y baja (restricción diastrática) y otras dos como propios del ambiente de los jóvenes (rasgo también diastrático).

De esto, claramente se puede concluir que estos saludos tienen indiscutibles restricciones de uso, mayormente de tipo diafásico y diastrático. Ahora bien, estas restricciones son comprensibles en el caso de los saludos 2 y 8 , por evidentes motivos del registro coloquial-informal (y hasta "pachuco") del vocablo "vara". Sin embargo, podemos preguntarnos: ¿Por qué motivo el resto de ellas también presentan restricciones pragmáticas o sociales, siendo que, para sus correlatos exactos utilizados "literalmente" como preguntas, aserciones o exclamaciones, en general nos podemos imaginar situaciones normales, y hasta formales, en las cuales no se consideren inapropiados aun dirigidos a personas con respecto a las cuales hay mucha distancia social? En otras palabras, ¿por qué esos saludos, dirigidos al jefe, a la profesora del curso al que se asiste o a alguien recientemente conocido, podrían ser percibidos por estos como inapropiados, en tanto que formulaciones idénticas, pero dirigidas a ellos en su interpretación literal (es decir, como preguntas, exclamaciones o aserciones) no lo serían?

Por el momento, voy a indicar que mi hipótesis es que las restricciones de uso de esos saludos, y sobre todo las diafásicas, tienen su origen, según ya mencioné, precisamente en el hecho de que sean saludos de apariencia fórica.
Parasermás específico,miplanteamientoes que la convencionalización de esas formulaciones para que puedan ser empleadas como saludos no incluye únicamente su interpretación como estrategias comunicativas de carácter fático $-\mathrm{y}$, por lo tanto, vacías de contenido proposicional, lo cual, tal como se mencionó en la introducción de este trabajo, es algo común a todos los saludossino también la instrucción tácita, igualmente de tipo pragmático, de que deben interpretarse como una ficción fórica. En otras palabras, que, aparte de compartir con cualquier otro tipo de saludo su carácter de fingimiento -por ejemplo, un ¿Cómo está? finge interesarse por el estado del otro, un Buenos días, finge expresar buenos deseos, y así sucesivamente- recurren a una ficción de segundo grado, en la medida en que fingen la existencia de un anclaje textual o referencial que no existe.

La idea es que, al emitir esos enunciados en calidad de saludos (es decir, en ausencia de un contexto discursivo que provea el anclaje endofórico, así como de un foco situacional que sirva como anclaje exofórico), el hablante presupone o da por sentada la existencia de una cercanía pragmática o social entre él y el receptor.

Esta presuposición lleva asociada la inferencia, también pragmática, de que, si hay una relativa cercanía o familiaridad entre él y el oyente, esto implica que en el pasado han tenido algún tipo de trato verbal o que, al menos, comparten determinados tipos de conocimientos, experiencias u otras características que los constituyen como pares.

Amparado en aquella presuposición de cercanía y en esta inferencia de paridad y experiencias compartidas, el hablante, al encontrarse en una situación comunicativa efectiva, se permite seleccionar y emitir un saludo que, por su forma, debería ser fórico, aun cuando su competencia comunicativa le indica que en realidad no es más que una ficción, pues, como ya se dijo, no existe en realidad un referente definido o unívocamente identificable al que apunte el eso de "¿Cómo va eso?", la cosa de "iAsí es la cosa!" y así sucesivamente. Sin embargo, el hablante no 
ve problema en hacer como si esos elementos deícticos o fóricos remitieran a referentes ya conocidos o identificables por el oyente (debido al supuesto de que son pares y por lo tanto comparten conocimientos y experiencias).

En este punto, el interlocutor tiene la opción de aceptar sin más el saludo, con todo y presuposición de familiaridad o cercanía incluidas, o dar alguna señal de que lo considera inapropiado, fundamentalmente porque rechaza la presuposición de paridad. Claro está, si esos enunciados los interpreta en su sentido literal, normalmente no tendría por qué considerar afectada su imagen social por recibirlos, independientemente de que la paridad refleje una situación real o no (con excepción de los casos 2 y 8 , pero esto por el registro del vocablo vara, que requiere de condiciones como identificación, solidaridad u otras para ser aceptable en un intercambio).

\section{Conclusión}

En síntesis, el empleo de los saludos seudofóricos, o de fingimiento fórico, encontraría su justificación en una presuposición de paridad o contigüidad social que serviría como fundamento a la ficción de que el receptor tiene los elementos suficientes (es decir, la información discursiva o situacional requerida) para identificar unívocamente el anclaje o el referente de los elementos fóricos implicados.

No hay que pensar, por supuesto, que los fundamentos de este tipo de estrategias sean manejados de forma consciente por hablantes y oyentes, sino que se emplean en la medida en que la producción e interpretación de estos recursos se encuentran institucionalizadas e integradas en la competencia comunicativa. Por último, hay que tomar en cuenta que esta clase de saludos podría contener más ejemplares que los aquí señalados, por lo cual los que aquí se han mencionado pueden tratarse, en principio y sin ningún problema, sencillamente como una muestra.
En la versión final de este trabajo trataré de fundamentar este planteamiento con base en algunos elementos conceptuales provistos por la Teoría de espacios mentales propuesta por Fauconnier (1994), ampliada por Fauconnier y Turner (2002), y sus desarrollos posteriores. En particular, será fundamental su concepto de espacios mezclados o combinados (blended spaces). En ese momento, además, se analizarán las particularidades de varias de las formulaciones estudiadas.

\section{Notas}

1. En términos más amplios, Haverkate (1994: 85) señala que: "La segunda categoría [la que se opone a la de los saludos monoléxicos] consta de fórmulas que literalmente especifican preguntas sobre la vida personal del intelocutor, particularmente sobre su salud o bienestar. Convencionalmente, estas preguntas se interpretan ante todo en un sentido simbólico; no se suele esperar una respuesta concreta a las mismas, lo cual queda sutilmente ilustrado por el adagio inglés: Don't tell your friends about your indigestion: "How are you!" is a greeting, not a question [No hables a tus amigos de tu indigestión: “CCómo estás?” es un saludo, no una pregunta]”.

2. Tal suposición tiene su base en la competencia comunicativa de quien esto escribe. La conveniencia de hacer una validación con informantes es sugerida por el hecho de que esa misma competencia señala a la posible existencia de restricciones de uso, por lo cual se decidió verificar ambos aspectos (empleo de esas formulaciones como saludos y existencia de restricciones de uso) con un cuestionario.

3. Nótese que los adverbios así y ahí pueden ser sustituidos sin problemas por construcciones con demostrativos: de esta/esa manera y en/a ese lugar, respectivamente (cf. DRAE, 2003: artículos correspondientes).

4. La hipótesis (o, tal vez, el postulado) de que la deixis espacial es más básica o primaria que las otras categorías deícticas, en las cuales se proyecta, es defendida por el mismo Yang (2011), quien específicamente afirma que "la deixis discursiva está basada en la concepción metafórica del discurso como tiempo, y del tiempo como espacio" (Yang, op. cit.: 134). 


\section{Bibliografía}

Cairns, Barbara. 1991. "Spatial deixis. The use of spatial co-ordinates in spoken language". Working Papers, 38: 19-28.

Fauconnier, Gilles. 1994. Mental spaces: Aspects of meaning construction in natural language. Cambridge y Nueva York: Cambridge University Press.

Fauconnier, Gilles y Mark Turner. 2002. The way we think: Conceptual blending and the mind's hidden complexities. Nueva York: Basic Books.

Haverkate, Henk. 1994. La cortesía verbal: estudio pragmalingüístico. Madrid: Gredos.
Levinson, Stephen C. 1983/1989. Pragmatics. Gran Bretaña: Cambridge University Press.

Real Academia Española. 2003. Diccionario de la lengua española [DRAE] (22 ${ }^{\mathrm{a}}$ ed.). Real Academia Española-Espasa Calpe. [Edición en CD-ROM, versión 1.0].

Rodríguez-Izquierdo y Gavala, Fernando. 1976. "La deixis anafórica en el artículo español. Comparación de textos de poesía y conversación". Revista española de lingüística, VI (1): 113-132.

Yang, Youwen. 2011. "A cognitive interpretation of discourse deixis". Theory and Practice in Language Studies, I (2): 128-135. 
